PROCEEDINGS OF THE

AMERICAN MATHEMATICAL SOCIETY

Volume 128, Number 6, Pages 1711-1720

S 0002-9939(99)05293-4

Article electronically published on October 27, 1999

\title{
ON THE DIVERGENCE OF THE $(C, 1)$ MEANS OF DOUBLE WALSH-FOURIER SERIES
}

\author{
G. GÁT \\ (Communicated by Christopher D. Sogge)
}

\begin{abstract}
In 1992, Móricz, Schipp and Wade proved the a.e. convergence of the double $(C, 1)$ means of the Walsh-Fourier series $\sigma_{n} f \rightarrow f\left(\min \left(n_{1}, n_{2}\right) \rightarrow\right.$ $\left.\infty, n=\left(n_{1}, n_{2}\right) \in \mathbb{N}^{2}\right)$ for functions in $L \log ^{+} L\left(I^{2}\right)\left(I^{2}\right.$ is the unit square). This paper aims to demonstrate the sharpness of this result. Namely, we prove that for all measurable function $\delta:[0,+\infty) \rightarrow[0,+\infty), \lim _{t \rightarrow \infty} \delta(t)=0$ we have a function $f$ such as $f \in L \log ^{+} L \delta(L)$ and $\sigma_{n} f$ does not converge to $f$ a.e. (in the Pringsheim sense).
\end{abstract}

\section{INTRODUCTION}

This paper is devoted to the problem of a.e. divergence of the $(C, 1)$ means of integrable functions with respect to the two-dimensional Walsh-Paley system. The problem of a.e. Cesàro summability is "quite delicate" in any local field setting (Taibleson, 6, p.114]). The dyadic case is no exception (see Fine [1], [2]). For double Walsh-Fourier series, Móricz, Schipp and Wade proved [4 that $\sigma_{\left(n_{1}, n_{2}\right)} f$ converges to $f$ a.e. in the Pringsheim sense (that is, no restriction on the indices $n_{1}, n_{2}$ other than $\left.\min \left(n_{1}, n_{2}\right) \rightarrow \infty\right)$ for all function $f$ in $L \log ^{+} L$. Since for the compact set $I^{2}$ (the unit square) the space $L \log ^{+} L$ is a proper subset of $L^{1}\left(I^{2}\right)$, then it is interesting to ask whether this theorem holds for $L^{1}\left(I^{2}\right)$ functions also? We give a negative answer. More precisely, we prove that the theorem of Móricz, Schipp and Wade cannot be sharpened. Namely, let $\delta:[0,+\infty) \rightarrow[0,+\infty)$ be a measurable function with property $\lim _{t \rightarrow \infty} \delta(t)=0$. We prove the existence of a function $f \in L^{1}\left(I^{2}\right)$ such as $f \in L \log ^{+} L \delta(L)$ (i.e., $\left.|f(x)| \log ^{+}(|f(x)|) \delta(|f(x)|) \in L^{1}\left(I^{2}\right)\right)$ and $\sigma_{\left(n_{1}, n_{2}\right)} f$ does not converge to $f$ a.e. as $\min \left(n_{2}, n_{2}\right) \rightarrow \infty$.

Let $\mathbb{P}$ denote the set of positive integers, $\mathbb{N}:=\mathbb{P} \cup\{0\}$, and $I:=[0,1)$. For any set $E$ let $E^{2}$ be the cartesian product $E \times E$. Thus $\mathbb{N}^{2}$ is the set of integral lattice points in the first quadrant and $I^{2}$ is the unit square. Let $E^{1}=E$ and fix $j=1$ or 2. Denote the $j$-dimensional Lebesgue measure $(\mu)$ of any set $E \subset I^{j}$ by $\mu(E)$. Denote the $L^{p}\left(I^{j}\right)$ norm of any function $f$ by $\|f\|_{p}(1 \leq p \leq \infty)$.

Denote the dyadic expansion of $n \in \mathbb{N}$ and $x \in I$ by $n=\sum_{j=0}^{\infty} n_{j} 2^{j}$ and $x=\sum_{j=0}^{\infty} x_{j} 2^{-j-1}$ (in the case of $x=\frac{k}{2^{m}} k, m \in \mathbb{N}$ choose the expansion which

Received by the editors July 13, 1998.

1991 Mathematics Subject Classification. Primary 42C10; Secondary 43A75, 40G05, 42B08.

Key words and phrases. Walsh group, double $(C, 1)$ means, divergence.

Research supported by the Hungarian National Foundation for Scientific Research (OTKA), grant no. F020334 and by the Hungarian "Müvelödési és Közoktatási Minisztérium", grant no. FKFP 0710/1997. 
terminates in zeros). $n_{i}, x_{i}$ are the $i$-th coordinates of $n, x$, respectively. Set $e_{i}:=1 / 2^{i+1} \in I$, the $i$ th coordinate of $e_{i}$ is 1 , the rest are zeros $(i \in \mathbb{N})$. Define the dyadic addition + as

$$
x+y=\sum_{j=0}^{\infty}\left(x_{j}+y_{j} \bmod 2\right) 2^{-j-1} .
$$

The sets $I_{n}(x):=\left\{y \in I: y_{0}=x_{0}, \ldots, y_{n-1}=x_{n-1}\right\}$ for $x \in I, I_{n}:=I_{n}(0)$ for $n \in$ $\mathbb{P}$ and $I_{0}(x):=I$ are the dyadic intervals of $I$. Denote $\mathcal{I}:=\left\{I_{n}(x): x \in I, n \in \mathbb{N}\right\}$; the elements of $\mathcal{I}$ are called the dyadic intervals on $I$. Denote by $\mathcal{A}_{n}$ the $\sigma$ algebra generated by the sets $I_{n}(x)(x \in I)$ and $E_{n}$ the conditional expectation operator with respect to $\mathcal{A}_{n}(n \in \mathbb{N})\left(f \in L^{1}\right)$. For $t=\left(t^{1}, t^{2}\right) \in I^{2}, b=\left(b_{1}, b_{2}\right) \in \mathbb{N}^{2}$ set the two-dimensional dyadic interval

$$
I_{b}^{2}(t):=I_{b_{1}}\left(t^{1}\right) \times I_{b_{2}}\left(t^{2}\right) .
$$

If $b \in \mathbb{N}$, then $I_{b}^{2}(t):=I_{b}\left(t^{1}\right) \times I_{b}\left(t^{2}\right)$. For $n=\left(n_{1}, n_{2}\right) \in \mathbb{N}^{2}$ denote by $E_{n}=E_{\left(n_{1}, n_{2}\right)}$ the two-dimensional expectation operator with respect to $\mathcal{A}_{n}=\mathcal{A}_{\left(n_{1}, n_{2}\right)}=\mathcal{A}_{n_{1}} \times$ $\mathcal{A}_{n_{2}}$. For $n \in \mathbb{N}$ denote $|n|:=\max \left(j \in \mathbb{N}: n_{j} \neq 0\right)$, that is, $2^{|n|} \leq n<2^{|n|+1}$. The Rademacher functions are defined as:

$$
r_{n}(x):=(-1)^{x_{n}} \quad(x \in I, n \in \mathbb{N}) .
$$

The Walsh-Paley system is defined as the set of Walsh-Paley functions:

$$
\omega_{n}(x):=\prod_{k=0}^{\infty}\left(r_{k}(x)\right)^{n_{k}}=(-1)^{\sum_{k=0}^{|n|} n_{k} x_{k}} \quad(x \in I, n \in \mathbb{N}) .
$$

That is, $\omega:=\left(\omega_{n}, n \in \mathbb{N}\right)$. Let us consider the Dirichlet and Fejér kernel functions:

$$
D_{n}:=\sum_{k=0}^{n-1} \omega_{k}, K_{n}:=\frac{1}{n} \sum_{k=1}^{n} D_{k}, D_{0}, K_{0}:=0 .
$$

The Fourier coefficients, the $n$-th partial sum of the Fourier series, the $n$-th $(C, 1)$ mean of $f \in L^{1}(I)$ :

$$
\begin{gathered}
\hat{f}(n):=\int_{I} f(x) \omega_{n}(x) d \mu(x)(n \in \mathbb{N}), \\
S_{n} f(y):=\sum_{k=0}^{n-1} \hat{f}(k) \omega_{k}(y)=\int_{I} f(x+y) D_{n}(x) d \mu(x)\left(n \in \mathbb{P}, S_{0} f=0\right), \\
\sigma_{n}(y):=\frac{1}{n} \sum_{k=1}^{n} S_{k}(y)=\int_{I} f(x+y) K_{n}(x) d \mu(x)\left(n \in \mathbb{P}, \sigma_{0} f=0, y \in I\right) .
\end{gathered}
$$

Define the two-dimensional Dirichlet and Fejér kernel functions as

$$
\begin{aligned}
D_{n}(x) & :=\sum_{0 \leq j_{1}<n_{1}, 0 \leq j_{2}<n_{2}} \omega_{j_{1}}\left(x^{1}\right) \omega_{j_{2}}\left(x^{2}\right)=D_{n_{1}}\left(x^{1}\right) D_{n_{2}}\left(x^{2}\right), \\
K_{n}(x) & :=\frac{1}{n_{1} n_{2}} \sum_{1 \leq j_{1} \leq n_{1}, 1 \leq j_{2} \leq n_{2}} D_{n}(x)=K_{n_{1}}\left(x^{1}\right) K_{n_{2}}\left(x^{2}\right),
\end{aligned}
$$


where $x=\left(x^{1}, x^{2}\right) \in I^{2}, n=\left(n_{1}, n_{2}\right) \in \mathbb{P}^{2}$ (for $n_{1} n_{2}=0$ set $\left.D_{n}=K_{n}=0\right)$. Also define the Fourier coefficients, the $n \in \mathbb{N}^{2}$-th partial sum of the Fourier series, the $n \in \mathbb{N}^{2}$-th $(C, 1)$ mean of $f \in L^{1}\left(I^{2}\right)$ :

$$
\begin{aligned}
& \hat{f}\left(\left(n_{1}, n_{2}\right)\right):=\int_{I^{2}} f\left(x^{1}, x^{2}\right) \omega_{n_{1}}\left(x^{1}\right) \omega_{n_{2}}\left(x^{2}\right) d \mu\left(x^{1}, x^{2}\right)\left(n \in \mathbb{N}^{2}\right), \\
& S_{\left(n_{1}, n_{2}\right)} f(y):=\sum_{k_{1}<n_{1}, k_{2}<n_{2}} \hat{f}\left(\left(k_{1}, k_{2}\right)\right) \omega_{k_{1}}\left(y^{1}\right) \omega_{k_{2}}\left(y^{2}\right) \\
&=\int_{I^{2}} f(x+y) D_{n}(x) d \mu(x)
\end{aligned}
$$

$\left(n \in \mathbb{P}^{2}, S_{n} f=0\right.$ for $\left.n_{1} n_{2}=0\right)$,

$$
\sigma_{\left(n_{1}, n_{2}\right)}(y):=\frac{1}{n_{1} n_{2}} \sum_{1 \leq k_{1} \leq n_{1}, 1 \leq k_{2} \leq n_{2}} S_{k}(y)=\int_{I^{2}} f(x+y) K_{n}(x) d \mu(x)
$$

$\left(n \in \mathbb{P}^{2}, \sigma_{n} f=0\right.$ for $\left.n_{1} n_{2}=0, y \in I^{2}\right)$.

\section{THE CONSTRUCTION}

Define a subset of the set of the two-dimensional intervalls $\mathcal{I} \times \mathcal{I}$ :

$$
\mathcal{I}_{n, a}(x):=\left\{I_{n+j}\left(x^{1}\right) \times I_{n+a-j}\left(x^{2}\right): j=0,1, \ldots, a\right\} \quad\left(x \in I^{2}, a, n \in \mathbb{N}\right) .
$$

It is easy to have

$$
\bigcap \mathcal{I}_{n, a}(x)=I_{n+a}\left(x^{1}\right) \times I_{n+a}\left(x^{2}\right), \quad \mu\left(\bigcap \mathcal{I}_{n, a}(x)\right)=2^{-2 n-2 a} ;
$$

$F \in \mathcal{I}_{n, a}(x)$ implies $\mu(F)=2^{-2 n-a}$. Next we prove

Lemma 1. $\mu\left(\bigcup \mathcal{I}_{n, a}(x)\right)=(1+a / 2) 2^{-2 n-a}$.

Proof. Denote (for the sake of this proof, only)

$$
\mu_{k}:=\mu\left(\bigcup_{j=0}^{k}\left(I_{n+j}\left(x^{1}\right) \times I_{n+a-j}\left(x^{2}\right)\right)\right)
$$

for $k=0,1, \ldots, a$. Then, $\mu_{0}=2^{-2 n-a}$ and for $k>0$ we have

$$
\begin{aligned}
\mu_{k} & =\mu_{k-1}+\mu\left(I_{n+k}\left(x^{1}\right) \times I_{n+a-k}\left(x^{2}\right)\right) \\
& -\mu\left(\bigcup_{j=0}^{k-1}\left(I_{n+j}\left(x^{1}\right) \times I_{n+a-j}\left(x^{2}\right)\right) \cap\left(I_{n+k}\left(x^{1}\right) \times I_{n+a-k}\left(x^{2}\right)\right)\right) \\
& =\mu_{k-1}+\frac{1}{2^{2 n+a}}-\mu\left(\bigcup_{j=0}^{k-1}\left(I_{n+k}\left(x^{1}\right) \times I_{n+a-j}\left(x^{2}\right)\right)\right) \\
& =\mu_{k-1}+\frac{1}{2^{2 n+a}}-\mu\left(I_{n+k}\left(x^{1}\right) \times I_{n+a-k+1}\left(x^{2}\right)\right) \\
& =\mu_{k-1}+\frac{1}{2^{2 n+a}}-\frac{1}{2^{2 n+a+1}}=\mu_{k-1}+\frac{1}{2^{2 n+a+1}} .
\end{aligned}
$$


This gives

$$
\begin{aligned}
\mu\left(\bigcup \mathcal{I}_{n, a}(x)\right) & =\mu\left(\bigcup_{j=0}^{a}\left(I_{n+j}\left(x^{1}\right) \times I_{n+a-j}\left(x^{2}\right)\right)\right)=\mu_{a} \\
& =\mu_{0}+a \frac{1}{2^{2 n+a+1}}=\frac{1}{2^{2 n+a}}+a \frac{1}{2^{2 n+a+1}}=\frac{1+a / 2}{2^{2 n+a}} .
\end{aligned}
$$

This completes the proof of Lemma 1.

For $t \in I^{2}, a, b, k \in \mathbb{N}$ define the sets $J_{a, b}^{k}(t), \Omega_{a, b}^{k}(t)$ recursively:

$$
J_{a, b}^{0}(t):=\{t\}, \quad \Omega_{a, b}^{0}(t):=\bigcup \mathcal{I}_{b, a}(t) .
$$

Suppose that the sets $J_{a, b}^{j}(t), \Omega_{a, b}^{j}(t)$ are defined for $j<k$. Then decompose

$$
I_{b}^{2}(t) \backslash \bigcup_{j=0}^{k-1} \Omega_{a, b}^{j}(t)
$$

as the disjoint union of dyadic squares of the form $I_{b+k a}^{2}(x)$. Take from each dyadic rectangle an element to represent. The set of $x$ 's corresponding to these squares is $J_{a, b}^{k}(t)$. That is,

$$
I_{b}^{2}(t) \backslash \bigcup_{j=0}^{k-1} \Omega_{a, b}^{j}(t)=\bigcup_{x \in J_{a, b}^{k}(t)} I_{b+k a}^{2}(x)
$$

Then, set

$$
\Omega_{a, b}^{k}(t):=\bigcup_{x \in J_{a, b}^{k}(t)} \bigcup \mathcal{I}_{b+k a, a}(x) .
$$

This gives the a.e. equality $I_{b}^{2}(t)=\bigcup_{j=0}^{\infty} \Omega_{a, b}^{j}(t)$. By this we get

$$
I^{2}=\bigcup_{\substack{t_{i}^{1}, t_{i}^{2} \in\{0,1\} \\ i=0,1, \ldots, b-1}} \bigcup_{j=0}^{\infty} \Omega_{a, b}^{j}(t)
$$

where $t=\left(t^{1}, t^{2}\right)=\left(t_{0}^{1} e_{0}+\ldots+t_{b-1}^{1} e_{b-1}, t_{0}^{2} e_{0}+\ldots+t_{b-1}^{2} e_{b-1}\right) \in I^{2}$. Set for $a, b, d \in \mathbb{N}(b \geq 2)$ the functions $\left(b^{\circ}:=[b / 2]-1([x]\right.$ denotes the integer part of $\left.x)\right)$ $f_{a, b}^{d}: I^{2} \rightarrow \mathbb{R}$ as follows:

$$
f_{a, b}^{d}(x):= \begin{cases}(-1)^{x_{b}^{1}+x_{b^{\circ}}^{2}} 2^{a}, & \text { if there exists } t \in I^{2}, k \leq d, y \in J_{a, b}^{k}(t) \\ & \text { for which } x \in \bigcap \mathcal{I}_{b+k a, a}(y), \\ 0, & \text { otherwise }\end{cases}
$$

Denoting by $1_{B}$ the characteristic set of any set $B \subset I^{2}$ we have

$$
f_{a, b}^{d}(x)=(-1)^{x_{b^{\circ}}^{1}+x_{b^{\circ}}^{2}} 2^{a} \sum_{\substack{t_{i}^{1}, t_{i}^{2} \in\{0,1\} \\ i=0,1, \ldots, b-1}} \sum_{k=0}^{d} \sum_{y \in J_{a, b}^{k}(t)} 1_{I_{b+k(a+1)}^{2}(y)}(x) .
$$

Lemma 2. For all $a, b, d \in \mathbb{N}$ we have $\int_{I^{2}}\left|f_{a, b}^{d}\right| \log ^{+}\left|f_{a, b}^{d}\right| \leq 2$. 
Proof.

$$
\begin{gathered}
\int_{I^{2}}\left|f_{a, b}^{d}(x)\right| \log ^{+}\left(\left|f_{a, b}^{d}(x)\right|\right) d \mu(x) \\
=2^{a} \log \left(2^{a}\right) \sum_{\substack{t_{i}^{1}, t_{i}^{2} \in\{0,1\} \\
i=0,1, \ldots, b-1}} \sum_{\substack{d \\
k=0}}^{d} \sum_{y \in J_{a, b}^{k}(t)} \mu\left(1_{I_{b+k(a+1)}^{2}(y)}(x)=1\right) \\
=2^{a} \log \left(2^{a}\right) \sum_{\substack{t_{i}^{1}, t_{i}^{2} \in\{0,1\} \\
i=0,1, \ldots, b-1}} \sum_{k=0}^{d} \sum_{y \in J_{a, b}^{k}(t)} \mu\left(\bigcap \mathcal{I}_{b+k a, a}(y)\right) \\
=2^{a} \log \left(2^{a}\right) \sum_{\substack{t_{i}^{1}, t_{i}^{2} \in\{0,1\} \\
i=0,1, \ldots, b-1}} \sum_{k=0}^{d} \sum_{y \in J_{a, b}^{k}(t)} \frac{\mu\left(\bigcup \mathcal{I}_{b+k a, a}(y)\right)}{2^{a}(1+a / 2)} \\
\leq \frac{\log \left(2^{a}\right)}{1+a / 2} \mu\left(I^{2}\right) \leq 2 .
\end{gathered}
$$

By the definition of the functions $f_{a, b}^{d}$ we have that

$$
f_{a, b}^{d}\left(.+e_{b^{\circ}}, .\right)=f_{a, b}^{d}\left(., .+e_{b^{\circ}}\right)=-f_{a, b}^{d}(., .) .
$$

It is known [5] p. 46] that for all $n \in \mathbb{P}, f \in L^{1}(I)$

$$
\sigma_{2^{n}} f(x)=\left(2^{n}+1 / 2\right) \int_{I_{n}(x)} f+\sum_{j=0}^{n-1} 2^{j-1} \int_{I_{n}(x)} f\left(.+e_{j}\right),
$$

by which we have for $n=\left(n_{1}, n_{2}\right) \in \mathbb{P}^{2}, x=\left(x^{1}, x^{2}\right) \in I^{2},\left(I_{n}^{2}(x)=I_{n_{1}}\left(x^{1}\right) \times\right.$ $\left.I_{n_{2}}\left(x^{2}\right)\right), f \in L^{1}\left(I^{2}\right)$

$$
\begin{aligned}
& \sigma_{\left(2^{n_{1}}, 2^{n_{2}}\right)} f(x)=\left(2^{n_{1}}+1 / 2\right)\left(2^{n_{2}}+1 / 2\right) \int_{I_{n}^{2}(x)} f(.) \\
& +\left(2^{n_{1}}+1 / 2\right) \sum_{j_{2}=0}^{n_{2}-1} 2^{j_{2}-1} \int_{I_{n}^{2}(x)} f\left(., .+e_{j_{2}}\right) \\
& +\left(2^{n_{2}}+1 / 2\right) \sum_{j_{1}=0}^{n_{1}-1} 2^{j_{1}-1} \int_{I_{n}^{2}(x)} f\left(.+e_{j_{1}}, .\right) \\
& +\sum_{j_{2}=0}^{n_{2}-1} \sum_{j_{1}=0}^{n_{1}-1} 2^{j_{1}+j_{2}-2} \int_{I_{n}^{2}(x)} f\left(.+e_{j_{1}}, .+e_{j_{2}}\right) .
\end{aligned}
$$

Lemma 3. Let $a, b, d \in \mathbb{P}, a-b^{\circ}<0\left(b^{\circ}=[b / 2]-1\right), b \geq 4$,

$$
x \in \bigcup_{\substack{t_{i}^{1}, t_{i}^{2} \in\{0,1\} \\ i=0,1, \ldots, b-1}} \bigcup_{k=0}^{d} \Omega_{a, b}^{k}(t) ;
$$


then there exists a unique $k \leq d$ for which

$$
x \in I_{b+k a+j}\left(y^{1}\right) \times I_{b+(k+1) a-j}\left(y^{2}\right),
$$

$y \in J_{a, b}^{k}(t)$. Setting $n:=(b+k a+j, b+(k+1) a-j) \in \mathbb{P}^{2}$ we have

$$
\left|\sigma_{\left(2^{n_{1}}, 2^{n_{2}}\right)} f_{a, b}^{d}(x)\right| \geq \frac{1}{4} .
$$

Proof. (2) is a straightforward consequence of the definition of $\Omega_{a, b}^{k}(t)$. Next, verify (3). Define $\tilde{f}_{a, b}^{d}$ by $f_{a, b}^{d}=(-1)^{x_{b}^{1}+x_{b}^{2} \circ} 2^{a} \tilde{f}_{a, b}^{d}$. Then $\tilde{f}_{a, b}^{d}$ is either 0 or 1 . Recall that

$$
\begin{gathered}
f_{a, b}^{d}\left(x^{1}, x^{2}+e_{j_{2}}\right)=(-1)^{x_{b}^{1} \circ}+x_{b}^{2} \circ 2^{a} \tilde{f}_{a, b}^{d}\left(x^{1}, x^{2}+e_{j_{2}}\right), \\
f_{a, b}^{d}\left(x^{1}+e_{j_{1}}, x^{2}\right)=(-1)^{x_{b}^{1}+x_{b}^{2}} 2^{a} \tilde{f}_{a, b}^{d}\left(x^{1}+e_{j_{1}}, x^{2}\right)
\end{gathered}
$$

for $j_{1}, j_{2} \neq e_{b^{\circ}}$ and

$$
f_{a, b}^{d}\left(.+e_{b^{\circ}}, .\right)=f_{a, b}^{d}\left(., .+e_{b^{\circ}}\right)=-f_{a, b}^{d}(., .) .
$$

By (1) we have

$$
\begin{aligned}
& \sigma_{\left(2^{n_{1}}, 2^{n_{2}}\right)} f_{a, b}^{d}(x)=2^{a}(-1)^{x_{b}^{1} \circ+x_{b^{\circ}}^{2}}\left(\left(2^{n_{1}}+1 / 2\right)\left(2^{n_{2}}+1 / 2\right) \int_{I_{n}^{2}(x)} \tilde{f}_{a, b}^{d}(.)\right. \\
& +\left(2^{n_{1}}+1 / 2\right) \sum_{j_{2}=0, j_{2} \neq b^{\circ}}^{n_{2}-1} 2^{j_{2}-1} \int_{I_{n}^{2}(x)} \tilde{f}_{a, b}^{d}\left(., .+e_{j_{2}}\right) \\
& +\left(2^{n_{2}}+1 / 2\right) \sum_{j_{1}=0, j_{1} \neq b^{\circ}}^{n_{1}-1} 2^{j_{1}-1} \int_{I_{n}^{2}(x)} \tilde{f}_{a, b}^{d}\left(.+e_{j_{1}, .}\right) \\
& \left.+\sum_{j_{2}=0, j_{2} \neq b^{\circ}}^{n_{2}-1} \sum_{j_{1}=0, j_{1} \neq b^{\circ}}^{n_{1}-1} 2^{j_{1}+j_{2}-2} \int_{I_{n}^{2}(x)} \tilde{f}_{a, b}^{d}\left(.+e_{j_{1}}, .+e_{j_{2}}\right)\right) \\
& -2^{a}(-1)^{x_{b}^{1} \circ+x_{b^{\circ}}^{2}}\left(\left(2^{n_{1}}+1 / 2\right)\left(2^{b^{\circ}-1}\right) \int_{I_{n}^{2}(x)} \tilde{f}_{a, b}^{d}(.)\right. \\
& \left.+\left(2^{n_{2}}+1 / 2\right)\left(2^{b^{\circ}-1}\right) \int_{I_{n}^{2}(x)} \tilde{f}_{a, b}^{d}(.)\right) \\
& -2^{a}(-1)^{x_{b}^{1} \circ+x_{b^{\circ}}}\left(\sum_{j_{2}=0, j_{2} \neq b^{\circ}}^{n_{2}-1} 2^{j_{2}+b^{\circ}-2} \int_{I_{n}^{2}(x)} \tilde{f}_{a, b}^{d}\left(., .+e_{j_{2}}\right)\right. \\
& \left.+\sum_{j_{1}=0, j_{1} \neq b^{\circ}}^{n_{1}-1} 2^{j_{1}+b^{\circ}-2} \int_{I_{n}^{2}(x)} \tilde{f}_{a, b}^{d}\left(.+e_{j_{1}}, .\right)\right) \\
& +2^{a}(-1)^{x_{b}^{1} \circ+x_{b^{\circ}}^{2}}\left(2^{b^{\circ}+b^{\circ}-2} \int_{I_{n}^{2}(x)} \tilde{f}_{a, b}^{d}(., .)\right) .
\end{aligned}
$$


From the above it follows that

$$
\begin{aligned}
& \left|\sigma_{\left(2^{n_{1}}, 2^{n_{2}}\right)} f_{a, b}^{d}(x)\right| \\
& \geq 2^{a+n_{1}+n_{2}} \int_{I_{n}^{2}(x)} \tilde{f}_{a, b}^{d}(.)-2^{a}\left(2^{n_{1}}+1 / 2\right)\left(2^{b^{\circ}-1}\right) \int_{I_{n}^{2}(x)} \tilde{f}_{a, b}^{d}(.) \\
& -2^{a}\left(2^{n_{2}}+1 / 2\right)\left(2^{b^{\circ}-1}\right) \int_{I_{n}^{2}(x)} \tilde{f}_{a, b}^{d}(.)-2^{a} \sum_{j_{2}=0, j_{2} \neq b^{\circ}}^{n_{2}-1} 2^{j_{2}+b^{\circ}-2} \int_{I_{n}^{2}(x)} \tilde{f}_{a, b}^{d}\left(., .+e_{j_{2}}\right) \\
& -2^{a} \sum_{j_{1}=0, j_{1} \neq b^{\circ}}^{n_{1}-1} 2^{j_{1}+b^{\circ}-2} \int_{I_{n}^{2}(x)} \tilde{f}_{a, b}^{d}\left(.+e_{j_{1}}, .\right) \\
& \geq 2^{a}\left(2^{n_{1}+n_{2}} \mu\left(I_{b+(k+1) a}\left(y^{1}\right) \times I_{b+(k+1) a}\left(y^{2}\right)\right)\right. \\
& -\left(\left(2^{n_{1}}+1 / 2\right)\left(2^{b^{\circ}-1}\right)+\left(2^{n_{2}}+1 / 2\right)\left(2^{b^{\circ}-1}\right)\right) \mu\left(I_{b+(k+1) a}\left(y^{1}\right) \times I_{b+(k+1) a}\left(y^{2}\right)\right) \\
& \left.-\left(\sum_{j_{2}=0, j_{2} \neq b^{\circ}}^{n_{2}-1} 2^{j_{2}+b^{\circ}-2}+\sum_{j_{1}=0, j_{1} \neq b^{\circ}}^{n_{1}-1} 2^{j_{1}+b^{\circ}-2}\right) \mu\left(I_{n}^{2}(x)\right)\right) \\
& \geq 2^{a}\left(2^{b+k a+j+b+(k+1) a-j} 2^{-2 b-2(k+1) a}-2^{b+(k+1) a+b^{\circ}+1} 2^{-2 b-2(k+1) a}\right. \\
& \left.-2^{b^{\circ}-2} 2^{-2 b-k a-(k+1) a}\left(\sum_{j_{1}=0}^{n_{1}-1} 2^{j_{1}}+\sum_{j_{2}=0}^{n_{2}-1} 2^{j_{2}}\right)\right) \geq \frac{1}{2} .
\end{aligned}
$$

Recall that $a<b^{\circ}=[b / 2]-1$. This completes the proof of Lemma 3 .

\section{The CONSTRUCTION OF THE FUnCTION}

Define the sequences $\left(\beta_{n}\right),\left(\delta_{n}\right),\left(a_{n}\right),\left(b_{n}\right),\left(d_{n}\right)$ recursively in the following way: $\left(\beta_{0}\right)=\left(\delta_{0}\right)=\left(a_{0}\right)=\left(b_{0}\right)=\left(d_{0}\right):=4$. For $n \in \mathbb{P}$

$$
\begin{aligned}
& \beta_{n}:=72 \max \left(n, \sum_{k=0}^{n-1} \beta_{k} 2^{a_{k}}\right), \\
& \delta_{n}:=\left[\sup \left\{t \in \mathbb{R}: \delta(t)>\frac{1}{2^{n} \beta_{n}}\right\}\right]+1\left(\text { if }\left\{t: \delta(t)>1 /\left(2^{n} \beta_{n}\right)\right\}=\emptyset, \text { then let } \delta_{n}=4\right), \\
& a_{n}:=\max \left(2^{n+1}, \delta_{n}, \log ^{+}\left(2 \beta_{n}\right)\right), \\
& b_{n}:=\max \left(b_{n-1}+\left(d_{n-1}+1\right) a_{n-1}+2,2 a_{n}\right) .
\end{aligned}
$$

By construction it is easy to see that the union of the disjoint sets $\Omega_{a, b}^{k}(t)(k \in \mathbb{N})$ is equal (neglecting a set of measure zero) to $I_{b}^{2}(t)$; hence there exists a $d_{n}$ for which

$$
\frac{1}{2^{2 b}}\left(1-\frac{1}{2^{n}}\right) \leq \mu\left(\bigcup_{k \leq d_{n}} \Omega_{a_{n}, b_{n}}^{k}(t)\right) \leq \frac{1}{2^{2 b}}
$$

Set

$$
G_{a_{n}, b_{n}, d_{n}}:=\bigcup_{\substack{t_{i}^{1}, t_{i}^{2} \in\{0,1\} \\ i=0,1, \ldots, b_{n}-1}} \bigcup_{j=0}^{d_{n}} \Omega_{a_{n}, b_{n}}^{j}(t)
$$

for all $n \in \mathbb{N}$ and $G:=\liminf G_{a_{n}, b_{n}, d_{n}}=\bigcup_{k \in \mathbb{N}} \bigcap_{n \geq k} G_{a_{n}, b_{n}, d_{n}}$. That is, $x \in I^{2}$ is an element of $G$ if and only if $x$ is an element of all but a finite number of 
$G_{a_{n}, b_{n}, d_{n}}$ 's. By (4) we have $1-\frac{1}{2^{n}} \leq \mu\left(G_{a_{n}, b_{n}, d_{n}}\right) \leq 1$, which easily gives that $\mu\left(I^{2} \backslash G\right)=0$.

Define the function $f$ as $f=\sum_{n=0}^{\infty} \beta_{n} f_{n}:=\sum_{n=0}^{\infty} \beta_{n} f_{a_{n}, b_{n}}^{d_{n}}$. At first we prove

Lemma 4. $\int_{I^{2}}|f(x)| \log ^{+}(|f(x)|) \delta(|f(x)|) d \mu(x)<\infty$.

Proof. Set

$$
H_{n}:=\left\{x \in I^{2}: f_{n}(x) \neq 0, f_{n+j}(x)=0(j \in \mathbb{P})\right\} \quad(n \in \mathbb{N})
$$

and $H_{-1}:=\left\{x \in I^{2}: f_{j}(x)=0(j \in \mathbb{P})\right\}$. The definiton of $f_{a, b}^{d},\left(a_{n}\right)$ gives

$$
\begin{aligned}
& \mu\left(H_{n}\right) \\
& \geq 1-\mu\left(\bigcup_{k>n}\left\{x \in I^{2}: f_{k}(x) \neq 0\right\}\right) \geq 1-\sum_{k>n} \mu\left(\left\{x \in I^{2}: f_{k}(x) \neq 0\right\}\right) \\
& \geq 1-\sum_{k>n} \frac{1}{2^{a_{k}}\left(a_{k} / 2+1\right)} \geq 1-\sum_{k>n} \frac{1}{2^{2^{k}}} .
\end{aligned}
$$

It follows that $\bigcup_{n=-1}^{\infty} H_{n}=I^{2}$ (neglecting a set of measure zero). Corresponding to this argument if $x \in H_{n}(n \in \mathbb{N})$, then

$$
\begin{aligned}
|f(x)| \leq \sum_{k=0}^{n-1} \beta_{k} 2^{a_{k}}+\beta_{n} 2^{a_{n}} \\
\leq \beta_{n}+\beta_{n} 2^{a_{n}} \leq 2 \beta_{n} 2^{a_{n}}=\left|\beta_{n} 2 f_{a_{n}, b_{n}}^{d_{n}}(x)\right|, \\
|f(x)| \geq \beta_{n} 2^{a_{n}}-\sum_{k=0}^{n-1} \beta_{k} 2^{a_{k}} \\
\geq \beta_{n} 2^{a_{n}}-\frac{1}{2} \beta_{n} \geq \frac{1}{2} \beta_{n} 2^{a_{n}} \\
=\frac{1}{2}\left|\beta_{n} f_{a_{n}, b_{n}}^{d_{n}}(x)\right| .
\end{aligned}
$$

Moreover, for $x \in H_{n}$ we have $|f(x)| \geq \frac{1}{2} \beta_{n} 2^{a_{n}} \geq 2^{a_{n}}>\delta_{n}$, which gives $\delta(|f(x)|) \leq$ $\frac{1}{2^{n} \beta_{n}}$. Consequently, by Lemma 2

$$
\begin{aligned}
& \int_{H_{n}}|f(x)| \log ^{+}(|f(x)|) \delta(|f(x)|) d \mu(x) \\
& \leq \int_{H_{n}} 2\left|\beta_{n} f_{a_{n}, b_{n}}^{d_{n}}(x)\right| \log ^{+}\left(2\left|f_{a_{n}, b_{n}}^{d_{n}}(x)\right|\right) \frac{1}{2^{n} \beta_{n}} \\
& \leq \int_{H_{n}} 2\left|\beta_{n} f_{a_{n}, b_{n}}^{d_{n}}(x)\right| \log ^{+}\left(\left|f_{a_{n}, b_{n}}^{d_{n}}(x)\right|^{2}\right) \frac{1}{2^{n} \beta_{n}} \\
& \leq \frac{4}{2^{n}} \int_{I^{2}}\left|f_{a_{n}, b_{n}}^{d_{n}}(x)\right| \log ^{+}\left(\left|f_{a_{n}, b_{n}}^{d_{n}}(x)\right|\right) d \mu(x) \leq \frac{8}{2^{n}} .
\end{aligned}
$$

Since for $x \in H_{-1}$ we have $f(x)=0$, then we get

$$
\begin{aligned}
& \int_{I^{2}}|f(x)| \log ^{+}(|f(x)|) \delta(|f(x)|) d \mu(x) \\
& \leq \sum_{n \in \mathbb{N}} \int_{H_{n}}|f(x)| \log ^{+}(|f(x)|) \delta(|f(x)|) d \mu(x) \leq 16 .
\end{aligned}
$$


Now, we are ready to prove

Theorem. For all measurable function $\delta:[0,+\infty) \rightarrow[0,+\infty), \lim _{t \rightarrow \infty} \delta(t)=0$ we have a function $f$ such that $f \in \operatorname{Llog}^{+} L \delta(L)$ and $\lim \sup \sigma_{\left(2^{n_{1}, 2^{n_{2}}}\right)} f(x)=+\infty$ $\left(\min \left(n_{2}, n_{2}\right) \rightarrow \infty\right)$, i.e. the two-dimensional $(C, 1)$ means does not converge to $f$ a.e. (in the Pringsheim sense).

It is not possible to prove more (e.g. to prove that $\lim \sigma_{\left(2^{n_{1}}, 2^{n_{2}}\right)} f(x)=+\infty$ $\left.\left(\min \left(n_{2}, n_{2}\right) \rightarrow \infty\right)\right)$, whereas Móricz, Schipp and Wade proved [4] for functions in $L^{1}\left(I^{2}\right)$ the a.e. convergence $\sigma_{\left(2^{n_{1}, 2^{n_{2}}}\right)} f \rightarrow f$ as the indices $n_{1}, n_{2} \rightarrow \infty\left(\left|n_{1}-n_{2}\right|\right.$ is bounded). The author in a different way proved [3] even more. Namely, for functions in $L^{1}\left(I^{2}\right)$ the a.e. convergence $\sigma_{\left(n_{1}, n_{2}\right)} f \rightarrow f$ holds as the indices $n_{1}, n_{2} \rightarrow$ $\infty$ restricted as $\beta^{-1} \leq n_{1} / n_{2} \leq \beta$ for some $\beta>1$ (compare with [7], 8], [9]).

Proof. We apply Lemmas 2,3 and 4. The rest is to prove the "divergence". Namely, we verify that for a.e. $x \in I^{2} \lim \sup \sigma_{\left(2^{n_{1}}, 2^{n_{2}}\right)} f(x)=+\infty\left(\min \left(n_{2}, n_{2}\right) \rightarrow \infty\right)$. This is the same as $\sup _{n_{1}, n_{2} \in \mathbb{N}} \sigma_{\left(2^{n_{1}}, 2^{\left.n_{2}\right)}\right.} f(x)=+\infty$. Let $x \in G$ (recall that $\mu\left(I^{2} \backslash G\right)=$ 0 ). Then there is an infinite number $n \in \mathbb{N}$ for which (even for all, but a finite number) $x \in G_{a_{n}, b_{n}, d_{n}}$. Then Lemma 3 gives that there exist a $t \in I^{2}, k \leq d_{n}$ for which $x \in \Omega_{a_{n}, b_{n}}^{k}(t)$, whereby, there are a $y \in J_{a_{n}, b_{n}}^{k}(t)$, a unique $j \in\left\{0,1, \ldots, a_{n}\right\}$ for which $x \in I_{b_{n}+k a_{n}+j}\left(y^{1}\right) \times I_{b_{n}+(k+1) a_{n}-j}\left(y^{2}\right)$. Set

$$
N:=\left(2^{N_{1}}, 2^{N_{2}}\right):=\left(b_{n}+k a_{n}+j, b_{n}+(k+1) a_{n}-j\right) \in \mathbb{P}^{2} .
$$

By Lemma 3 we have

$$
\left|\sigma_{\left(2^{\left.N_{1}, 2^{N_{2}}\right)}\right.} f_{a_{n}, b_{n}}^{d_{n}}(x)\right| \geq \frac{1}{2} .
$$

In [5] p. 46] one can find that $\left\|K_{n}\right\|_{1} \leq 3$ for $n \in \mathbb{N}$ which in the standard way (see e.g. [5]) gives $\left\|\sigma_{n} f\right\|_{\infty} \leq 9\|f\|_{\infty}\left(f \in L^{1}\left(I^{2}\right), n \in \mathbb{P}^{2}\right)$. It follows for $i \in \mathbb{P}$ that

$$
\left\|\sigma_{\left(2^{\left.N_{1}, 2^{N_{2}}\right)}\right.} f_{a_{n-i}, b_{n-i}}^{d_{n-i}}\right\|_{\infty} \leq 9\left\|f_{a_{n-i}, b_{n-i}}^{d_{n-i}}\right\|_{\infty} \leq 9 \cdot 2^{a_{n-i}}
$$

The definition of function $f_{a, b}^{d}$ gives that $E_{\left(b^{\circ}, b^{\circ}\right)} f_{a, b}^{d}=0$ and also that for $j_{1}, j_{2}<b^{\circ}$ natural numbers $E_{\left(b^{\circ}, b^{\circ}\right)} f_{a, b}^{d}\left(.+e_{j_{1}},.\right)=E_{\left(b^{\circ}, b^{\circ}\right)} f_{a, b}^{d}\left(., .+e_{j_{1}}\right)=0$. Hence, for $k \in \mathbb{P}$ it follows that

$$
\begin{aligned}
& 2^{N_{1} N_{2}} \int_{I_{N}^{2}(x)} f_{a_{n+k}, b_{n+k}}^{d_{n+k}}\left(.+e_{j_{1}}, .\right) \\
& =E_{\left(N_{1}, N_{2}\right)} f_{a_{n+k}, b_{n+k}}^{d_{n+k}}\left(.+e_{j_{1}}, .\right)(x) \\
& =E_{\left(N_{1}, N_{2}\right)}\left(E_{\left(b_{n+k}^{\circ}, b_{n+k}^{\circ}\right)} f_{a_{n+k}, b_{n+k}}^{d_{n+k}}\left(.+e_{j_{1}}, .\right)\right)(x)=0
\end{aligned}
$$

and

$$
\begin{aligned}
& 2^{N_{1} N_{2}} \int_{I_{N}^{2}(x)} f_{a_{n+k}, b_{n+k}}^{d_{n+k}}\left(., .+e_{j_{2}}\right) \\
& =E_{\left(N_{1}, N_{2}\right)} f_{a_{n+k}, b_{n+k}}^{d_{n+k}}\left(., .+e_{j_{2}}\right)(x) \\
& =E_{\left(N_{1}, N_{2}\right)}\left(E_{\left(b_{n+k}^{\circ}, b_{n+k}^{\circ}\right)} f_{a_{n+k}, b_{n+k}}^{d_{n+k}}\left(.,+e_{j_{2}}\right)\right)(x)=0 .
\end{aligned}
$$

This gives that for all $k \in \mathbb{P}$

$$
\sigma_{\left(2^{\left.N_{1}, 2^{N_{2}}\right)}\right.} f_{a_{n+k}, b_{n+k}}^{d_{n+k}}(x)=0
$$


Consequently,

$$
\begin{aligned}
& \left|\sigma_{\left(2^{\left.N_{1}, 2^{N_{2}}\right)}\right.} f(x)\right| \\
& \geq\left|\sigma_{\left(2^{N_{1}}, 2^{N_{2}}\right)} \beta_{n} f_{a_{n}, b_{n}}^{d_{n}}(x)\right|-\sum_{i=1}^{n-1}\left|\sigma_{\left(2^{N_{1}}, 2^{N_{2}}\right)} \beta_{i} f_{a_{i}, b_{i}}^{d_{i}}(x)\right| \\
& \geq \frac{1}{2} \beta_{n}-9 \sum_{i=0}^{n-1} \beta_{i}\left\|f_{a_{i}, b_{i}}^{d_{i}}\right\|_{\infty} \\
& \geq \frac{1}{2} \beta_{n}-9 \sum_{i=0}^{n-1} \beta_{i} 2^{a_{i}} \geq \frac{1}{4} \beta_{n} \geq 18 n .
\end{aligned}
$$

This completes the proof of the theorem.

\section{REFERENCES}

1. N.J. Fine, On the Walsh functions, Trans Amer. Math. Soc. 65 (1949), 372-414.

2. Cesàro summability of Walsh-Fourier series, Proc. Nat. Acad. Sci. USA 41 (1955), 558-591. MR 17:31f

3. G. Gát, Pointwise convergence of double Walsh-Fejér means, Annales Univ. Sci. Budapestiensis, Sect. Comp. 16 (1996), 173-184.CMP 97:14

4. F. Móricz, F. Schipp, and W.R. Wade, Cesàro summability of double Walsh-Fourier series, Trans Amer. Math. Soc. 3291 (1992), 131-140. MR 92j:42028

5. F. Schipp, W.R. Wade, P. Simon, J. Pál, Walsh series," An Introduction to dyadic harmonic analysis", Adam Hilger, Bristol and New York, 1990. MR 92g:42001

6. M.H. Taibleson, Fourier Analysis on Local Fields, Princeton Univ. Press., Princeton ,N.J., 1975. MR 58:6943

7. F. Weisz, Cesàro summability of two-dimensional Walsh-Fourier series, Trans. Amer. Math. Soc. 348 (1996), 2169-2181. MR 96i:42004

8. F. Weisz, Cesàro summability of one- and two-dimensional Walsh-Fourier series, Analysis Math. 22 (1996), 229-242. CMP 98:14

9. F. Weisz, Cesàro summability of two-parameter Walsh-Fourier series, J. Appr. Theory 88 (1997), 168-192. MR 98e:42024

10. A. Zygmund, "Trigonometric series", Cambridge University Press, New York, N.Y., 1959.

Department of Mathematics, Bessenyei College, Nyíregyháza, P.O. Box 166., H-4400, HUNGARY

E-mail address: gatgy@agy.bgytf.hu 